\title{
How do synapses measure milliseconds?
}

\section{Thomas G. Oertner*}

Friedrich Miescher Institute for Biomedical Research, Basel, Switzerland

*Correspondence: thomas.oertner@fmi.ch

\section{A commentary on}

Changes of the EPSP waveform regulate the temporal window for spike-timingdependent plasticity.

Fuenzalida, M., Fernandez de Sevilla, D., and Buño, W. (2007). J. Neurosci. 27, 11940-11948

and

Kinetics of $\mathrm{Mg}^{2+}$ unblock of NMDA receptors: implications for spike-timing dependent synaptic plasticity.

Kampa, B. M., Clements, J., Jonas, P., and Stuart, G. J. (2004). J. Physiol. 556, 337-345.

Experimental validation of a theoretical prediction by measurements has often produced a critical step forward in our understanding of brain function. Donald Hebb's original idea that the repetitive contribution of cell A to cell Bs firing should result in a strengthening of this particular connection was spectacularly confirmed by the discovery of spike timing dependent plasticity (STDP; Bi and Poo, 1998; Markram et al., 1997). This finding attracted immediate attention, since plasticity mechanisms based on spike timing seemed much more relevant physiologically than protocols that were based on high frequency tetanization. Mechanistically, the NMDA receptor, through its sensitivity to both glutamate and membrane potential, seemed ideally poised to detect the timing between presynaptic release and backpropagating action potentials (bAPs). In consequence, most modeling studies have focused exclusively on NMDAR properties and downstream mechanisms to explain synaptic coincidence detection.

However, new experimental data suggest that this textbook view of coincidence detection is too reductionist. First of all, the time course of NMDAR activity does not fit very well the length of the potentiation window. Due to the slow off-rate for glutamate, NMDAR currents decay with a time constant of $\sim 100 \mathrm{~ms}$, too slow to explain the 10-20 ms window for induction of longterm potentiation by STDP (Clements et al., 1992; Franks and Sejnowski, 2002; Sjostrom et al., 2008). This contradiction was only partially alleviated by the discovery that $\mathrm{Mg}^{2+}$ ions do not only block NMDARs, but also reduce their affinity for glutamate and enhance desensitization. The half duration of NMDAR activation at physiological $\mathrm{Mg}^{2+}$ concentrations has been estimated to be 43 ms (Kampa et al., 2004), significantly shorter than previous estimates, but still too long to explain the size of the STDP window (Figure 1).A potential solution was sketched out many years ago, based on the idea that dendritic spines could act as electrical amplifiers during the EPSP (Shepherd, 1996). Due to positive feedback provided by high-threshold voltage-gated conductances, spines could be exquisitely sensitive to additional depolarization during the EPSP. After the EPSP, on the other hand, bAPs would not provide sufficient depolarization to activate the spine amplifier, even if many NMDARs were still bound to glutamate. In this view, the entire spine acts as the coincidence detector (Shepherd, 1996). The window for

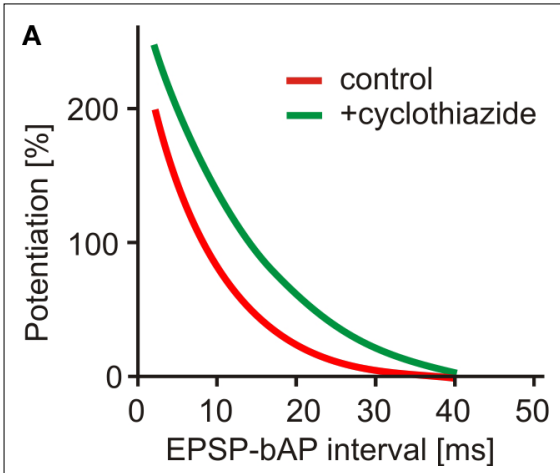

STDP would then be set by the amplitude and duration of the spine EPSP, which is primarily a function of the AMPAR current and the input resistance of the spine. In a recent study (Fuenzalida et al., 2007), it has been demonstrated that the STDP window can be significantly extended by prolonging AMPAR currents (Figure 1A). In the same study, increasing membrane conductance by the activation of $\mathrm{K}^{+}$channels resulted in a narrowing of the STDP window. This is consistent with the idea that a decreased input resistance reduces the EPSP inside the spine and thus shortens the activation of the spine amplifier.

A parameter of critical importance for both amplitude and duration of the spine EPSP is the electrical resistance of the spine neck (Segev and Rall, 1988; Tsay and Yuste, 2004). Unfortunately, it is very difficult to measure spine head depolarization, for example by voltage imaging. Since the resistivity of the cytoplasm inside the spine neck is not known, resistance estimates from compartmental modeling are also not very reliable. We have recently measured calcium transients in synaptically stimulated spines and found a positive correlation between NMDA receptor unblocking and diffusional resistance of the spine neck, pointing to a high degree

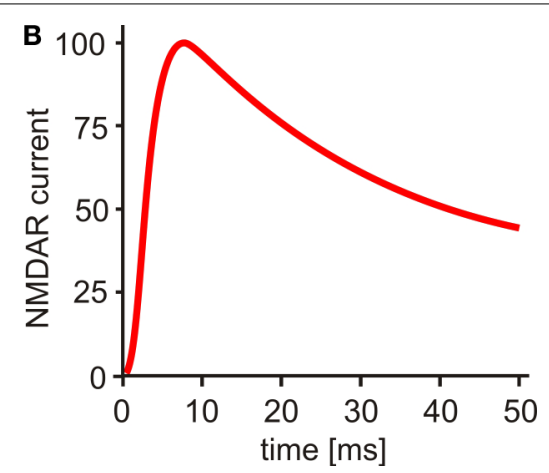

FIGURE 1 | (A) Spike timing dependent plasticity: LTP after EPSP-AP pairing. Boosting AMPA receptor currents with cyclothiazide (green curve) prolongs the window for LTP induction (adapted from Fuenzalida et al., 2007). (B) NMDA receptor current at physiological $\mathrm{Mg}^{2+}$ concentration outlasts the STDP window (adapted from Kampa et al., 2004). 
of electrical isolation in a subset of spines (Grunditz et al., 2008). Thus, a new picture emerges, in which structural features of the spine as well as its complement of voltage- and ligand-gated channels have to be considered to understand the exquisite sensitivity of synapses to the precise timing of pre- and postsynaptic activity. Just as the time constant of an RC circuit can not be understood by investigating the resistor or capacitor in isolation, coincidence detection is a function of the spine as a whole. A possibility to be tested is that only synapses on spines are sensitive to STDP protocols. If this prediction holds true, activitydependent changes in spine neck resistance could regulate plasticity at the level of individual synapses (Bloodgood and Sabatini, 2005). In any case, the sluggish response of NMDARs provides a poor explanation for the fact that synapses apparently can sense the timing of electrical events with millisecond precision. While NMDARs are key channels for calcium entry, they have to be brought up to the steep part of their activation curve by AMPAR activity. Only under these conditions are they sensitive to the small additional depolarization provided by bAPs. The fast kinetics of AMPARs in combination with a high spine neck resistance is the secret behind the tight temporal control of biochemical signaling at spine synapses.

\section{REFERENCES}

Bi, G. Q., and Poo, M. M. (1998). Synaptic modifications in cultured hippocampal neurons: dependence on spike timing, synaptic strength, and postsynaptic cell type. J. Neurosci. 18, 10464-10472.

Bloodgood, B. L., and Sabatini, B. L. (2005). Neurona activity regulates diffusion across the neck of dendritic spines. Science 310, 866-869.

Clements, J. D., Lester, R. A., Tong, G., Jahr, C. E., and Westbrook, G. L. (1992). The time course of glutamate in the synaptic cleft. Science 258, 1498-1501.

Franks, K. M., and Sejnowski, T. J. (2002). Complexity of calcium signaling in synaptic spines. Bioessays 24 , 1130-1144.

Fuenzalida, M., Fernandez de Sevilla, D., and Buno, W. (2007). Changes of the EPSP waveform regulate the temporal window for spike-timing-dependent plasticity. J. Neurosci. 27, 11940-11948.

Grunditz, A., Holbro, N., Tian, L., Zuo, Y., and Oertner, T. G. (2008). Spine neck plasticity controls postsynaptic calcium signals through electrical compartmentalization. J. Neurosci. 28, 13457-13466.
Kampa, B. M., Clements, J., Jonas, P., and Stuart, G. J. (2004). Kinetics of $\mathrm{Mg}^{2+}$ unblock of NMDA receptors: implications for spike-timing dependent synaptic plasticity. J. Physiol. 556, 337-345.

Markram, H., Lübke, J., Frotscher, M., and Sakmann, B. (1997). Regulation of synaptic efficacy by coincidence of postsynaptic APs and EPSPs. Science 275, 213-215.

Segev, I., and Rall, W. (1988). Computational study of an excitable dendritic spine. J. Neurophysiol. 60 , 499-523.

Shepherd, G. M. (1996). The dendritic spine: a multifunctional integrative unit. J. Neurophysiol. 75, 2197-2210.

Sjostrom, P. J., Rancz, E. A., Roth, A., and Hausser, M. (2008). Dendritic excitability and synaptic plasticity. Physiol. Rev. 88, 769-840.

Tsay, D., and Yuste, R. (2004). On the electrical function of dendritic spines. Trends Neurosci. 27, 77-83.

Received: 27 January 2009; published online: 27 April 2009.

Citation: Oertner TG (2009) How do synapses measure milliseconds? Front. Comput. Neurosci. 3:7. doi: 10.3389/neuro.10.007.2009

Copyright (C) 2009 Oertner. This is an open-access publication subject to an exclusive license agreement between the authors and the Frontiers Research Foundation, which permits unrestricted use, distribution, and reproduction in any medium, provided the original authors and source are credited. 